\title{
A contour extraction algorithm based on wavelet transform and dynamic contour compensation
}

\author{
Bao-Yan $\mathrm{Mu}^{\uparrow}$, Shuai Chen and Yue Ma \\ Shenyang Institute of Automation Chinese Academy of Sciences \\ Shenyang, Liaoning, China \\ 'E-mail:mubaoyan@sia.cn
}

\begin{abstract}
Contour extraction process is often disturbed by complex backgrounds or foregrounds in the machine vision system. A new contour extraction algorithm is proposed based on wavelet transform and dynamic contour compensation. The wavelet transform method is used to extract the edge of image, and the dynamic compensation is used to restore the contour which is affected by the complex dark areas such as bar code or black icon. The algorithm is applied in a sausage machine vision inspection system, and it is proved effective in eliminating the contour depression and retaining the actual defect features.
\end{abstract}

Keywords: Edge Detection; Wavelet Transform; Contour Compensation.

\section{General Instructions}

In order to ensure the quality of sausage, sausage products are artificial inspection in the domestic factories. Because the inspection work is single, tedious and repetitive, the workers are easy to be affected by many external factors. Along with the development of our country economy, labor costs are becoming more and more high. In order to improve the product quality and reduce the production costs, the sausage online inspection machine vision system is developed. The market for the devices used to automatic picking sausages has a very urgent needs.

The device can detect a variety of sausage defects including bubble, nip, clasp, rib head and cut. The bubble is one of the most common defects of the sausage in the packaging process, which usually appears in the joint of sausage casing. Sausage edge extraction is a very important step in the visual detection, and the quality of the edge extraction directly determines the final detection results. Accurate edge extraction can help us to extract the complete image of sausage from the collected pictures and ensure the analysis and research of the next step.

The difference of sausage casing color is very large, and it is easily disturbed by noise, so the traditional differential method and threshold method 
used in extracting the sausage edge do not play well[1]. While wavelet transform has good time-frequency characteristics, it can suppress the interference and get the accurate edge. Sometimes there will be some dark areas in sausage casing, at this time the extracted edge will be depressed. This will affect the calculation of the contour, which does not conform to the actual situation. Dynamic contour compensation algorithm can not only compensate contour depression, but also keep defect features as much as possible.

\section{Edge Detection Based on Wavelet Transform}

Wavelet transform is a time-frequency analysis method of signals with the feature of multi-resolution analysis. It has the ability of showing local features of the signal both in the time domain and frequency domain. With the multiresolution analysis of wavelet transform, noise can be suppressed in large scale, and the precise positioning of the edge can be achieved in small scale[2].

\subsection{The principle of wavelet transform}

The basic idea of edge detection based on wavelet transform is choosing the first derivative or two order derivative of the smoothing function as the wavelet function first. Then apply wavelet transform to the image signal and calculate wavelet transform modulus maxima. Supposed that $\theta(x, y)$ is a two-dimensional smoothing function, and meet the following condition, as in Eq. (1).

$$
\int_{-\infty}^{\infty} \int_{-\infty}^{\infty} \theta(x, y) d x d y=1, \lim _{x^{2}+y^{2} \rightarrow \infty} \theta(x, y)=0 .
$$

The definition of $\theta_{s}(x, y)$ is as in Eq. (2):

$$
\theta_{s}(x, y)=\frac{1}{s^{2}} \theta\left(\frac{x}{s}, \frac{y}{s}\right) .
$$

Wavelet function $\psi^{1}(x, y), \psi^{2}(x, y)$ can be defined as followed:

$$
\left\{\begin{array}{l}
\psi^{1}(x, y)=\frac{\partial \theta(x, y)}{\partial x} \\
\psi^{2}(x, y)=\frac{\partial \theta(x, y)}{\partial y}
\end{array}\right.
$$

Then, wavelet function is used to carry out the wavelet transform. According to the definitions of $\psi^{1}(x, y), \psi^{2}(x, y)$, the wavelet transform is defined as in Eq. (4): 


$$
\left[\begin{array}{c}
W_{s}^{1} f(x, y) \\
W_{s}^{2} f(x, y)
\end{array}\right]=\left[\begin{array}{l}
f * \psi_{s}^{1}(x, y) \\
f * \psi_{s}^{2}(x, y)
\end{array}\right]=s\left[\begin{array}{l}
\frac{\partial}{\partial x} f * \theta_{s}(x, y) \\
\frac{\partial}{\partial y} f * \theta_{s}(x, y)
\end{array}\right]=s \nabla f * \theta_{s}(x, y) .
$$

$s \nabla f * \theta_{s}(x, y)$ is a gradient vector. Supposed that the scale parameter is $s=\left\{2^{j}\right\}_{j \in Z}$, then two-dimensional function sequence $W f$ can be called twodimensional dyadic wavelet transform of $f(x, y)$ :

$$
W f=\left\{W_{2^{j}}^{1} f(x, y), W_{2^{j}}^{2} f(x, y)\right\}_{j \in Z}
$$

When $s=2^{j}$, calculate the value of module and the angle between the gradient vector and the horizontal direction:

$$
\left\{\begin{array}{l}
M_{2^{j}} f(x, y)=\sqrt{\left|W_{2^{j}}^{1} f(x, y)\right|^{2}+\left|W_{2^{j}}^{2} f(x, y)^{2}\right|} \\
A_{2^{j}} f(x, y)=\arg \left(W_{2^{j}}^{1} f(x, y)+i W_{2^{j}}^{2} f(x, y)\right)
\end{array} .\right.
$$

Obviously, the local maximum point of module $M_{2^{j}} f(x, y)$ corresponds to mutation point of function $f^{*} \theta_{s}(x, y)$, and $A_{2^{j}} f(x, y)$ shows the direction of gradient. The choice of scale $s$ is very important in multi-scale edge detection[3]. As the scale increases, the image becomes smoother, the high frequency noise is more suppressed, and signal-to-noise ratio of the image is improved, but at the same time, the edge of image gets coarsening, positioning accuracy is decreased. On the other hand, when the scale becomes smaller, the signal-to-noise ratio of the image is reduced, the reliability of image edge detected, but the accuracy is increased.

According to the above analysis, an effective edge detection algorithm is designed, which can restrain the noise of the original image with a large scale, and the edge of the image can be precisely located by the small scale filter. Therefore, multi-scale wavelet transform can be used to detect the edge points of the image accurately[4].

\subsection{The detection results of sausage edge}

There is a lot of interference such as the meat mud and other impurities on the sorting belt, bad subtraction of background template and the jitter of camera and the light source in the process of collecting sausage image with camera. In the presence of these interferences, using the original threshold method and the differential method, the edge of image will contain a burr or bump, which is inconsistent with the actual situation. This will seriously affect the detection 
result. If using the wavelet transform mentioned above, noise interference can be eliminated, and the accurate edge of sausage can be extracted.

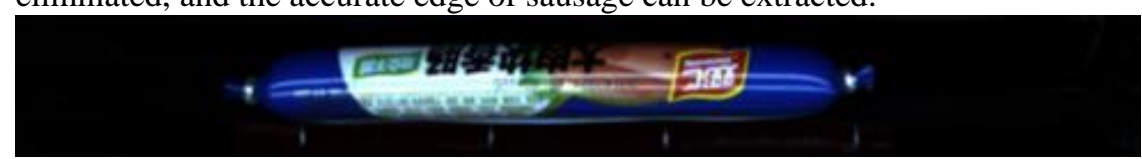

Fig. 1. The image after background subtraction.

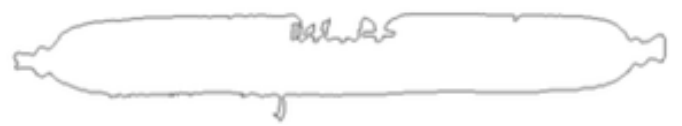

Fig. 2. The edge obtained using the method of threshold method.

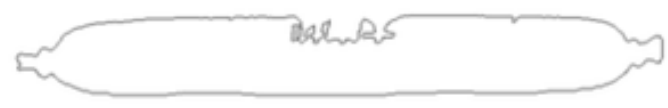

Fig. 3. The edge obtained using the method of wavelet transform

Fig. 1 shows the image of sausage after background subtraction. There is noise and interference caused by background removal in this image. Fig. 2 is the edge obtained with threshold method. There is burr and bump on the edge in the image, which is obviously a wrong result. Fig. 3 is the edge obtained with wavelet transform method. The edge of image is relatively smooth without considering the effects of the black areas in casing in Fig. 3. This edge is more close to the actual situation.

\section{Dynamic Contour Compensation Algorithm}

The bubble at the joint of sausage is convex, but the interference caused by the color darker areas such as bar code and black icon in casing is concave. So a contour compensation algorithm is designed to compensate contour depression and keep defect features as much as possible. The main contour of casing is close to a straight line, for normal goods, using Hough Transform and least square fitting method, the edge close to the actual contour line can be achieved. When there is bubble defects and black icon in casing, although this kind of method can compensate error edge, but will lose valuable information on convex casing.

In this case, a dynamic contour compensation algorithm is designed, and this algorithm can not only remove the small depression generated by the black Icon[5], but also completely retain the characteristics of the bubble and bending.

\subsection{The principle of dynamic contour compensation algorithm}

Dynamic contour compensation algorithm is derived from contour convex hull method. Convex hull is the connection between contour convex points. Dynamic 
contour compensation algorithm is calculating the outer circle trajectory of the detected edge points. In the process of calculation trajectory, circle radius is dynamic, it is influenced by the vertical position of the edge points.

Supposed that the edge points detected are $\left(x_{1}, y_{1}\right),\left(x_{2}, y_{2}\right) \cdots\left(x_{n}, y_{n}\right)$ in turn in the Fig. 4. The center coordinate is $\left(x_{p}, y_{p}\right)$, and the center $\left(x_{p}, y_{p}\right)$ is at the top of the edge point $\left(x_{q}, y_{q}\right)$, that is $x_{p}=x_{q} .\left(x_{p}, y_{p}\right)$ meets the following formula:

$$
\min \left\{\sqrt{\left(x_{p}-x_{i}\right)^{2}+\left(\mathrm{y}_{p}-\mathrm{y}_{i}\right)^{2}}\right\}=R
$$

Here, $i=(q-R),(\mathrm{q}-R+1) \cdots(q+R-1),(q+R)$. The coordinate $y_{p}$ can be obtained by using the above formula. The coordinate of contour points obtained by compensation is $\left(\mathrm{x}_{p}, \mathrm{y}_{p}-R\right)$.

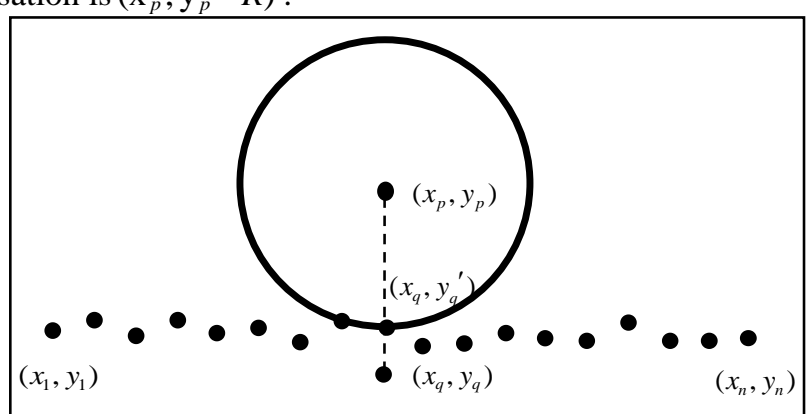

Fig. 4. Principle diagram of dynamic contour compensation algorithm.

The radius $R$ is dynamic, it is affected by the depth and width of sag. Supposed that $\alpha$ is sag depth factor, $\beta$ is sag width factor. DisMax is Maximum value of the difference between the mean of vertical coordinates of all edge points and vertical coordinates of the edge points under the outer circle. DisSum is sum of the mean of vertical coordinates of all edge points and vertical coordinates of the edge points under the outer circle. The larger the impact factor, the more the compensation for contour depression. The smaller the impact factor, the more complete the characteristics of bubble. In the process of calculating contour, the value of the outer circle radius will be affected by sag depth and width[6]. The radius $R$ meets the following formula:

$$
R=\alpha^{*} \text { DisMax }+\beta^{*} \text { DisSum. }
$$

Here, if DisMax $<0$, then $\alpha=0$. If DisSum $<0$, then $\beta=0$.If DisMax $<0$ and DisSum $<0$, then $R=0$. When $R=0$, It is indicated that there is no sag in the area below circle, so it is not necessary to carry out the contour compensation at this time. 


\subsection{The results of dynamic contour compensation}

The black area in sausage casing shown in Fig.5 is very obvious, the edge obtained with the above algorithm is depressed. Dynamic contour compensation algorithm is designed to compensate the contour. Fig. 6 is the result after contour compensation when $R=20$. Fig. 7 is the result after contour compensation when $R=50$. Fig. 8 is the result after contour compensation when $R=100$.From the results, it can be seen that the greater the radius of the outer circle, the larger the degree of compensation for depression, but at the same time, it will also reduce the salient features.

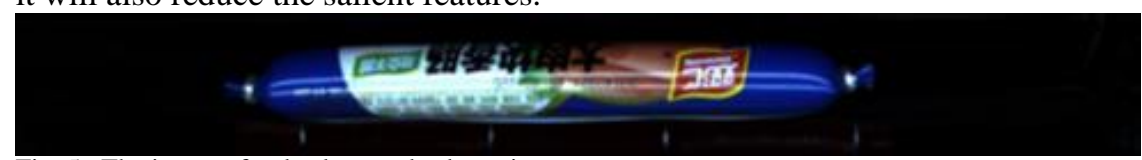

Fig. 5. The image after background subtraction.

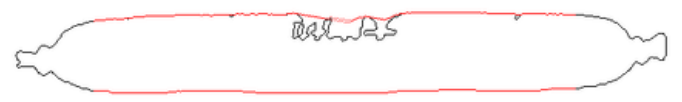

Fig. 6. The result after contour compensation when $R=20$.

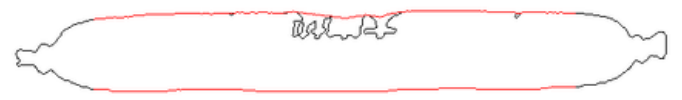

Fig. 7. The result after contour compensation when $R=50$.

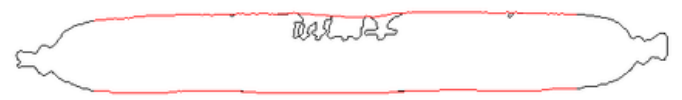

Fig. 8. The result after contour compensation when $R=100$.

\section{Conclusions}

This paper provides a contour extraction algorithm used in the visual inspection system. First, the edge of sausage is extracted by the method of wavelet transform. Second, the dynamic contour compensation algorithm is applied to modify the edge. The algorithm can not only eliminate the defect, but also retain the characteristics of the defect. Through the image processing proposed above, sausage online inspection machine system can inspect the defects of sausage accurately.

\section{References}

1. Shumin Liu, Xujuan Xu, Yingping Huang. Anti-noise Snake model and application in pedestrian contour extraction. Information and Control, 2015, 44(5): 564-569. 
2. Dinc E, Baleanu D. New approaches for simultaneous spectral analysis of a complex mixture using the fractional wavelet transform. Commun Nonlinear Sci Numer Simul, 2010, 15: 812-818

3. Xia Zhang, Weichao Sun and Tong Shuai, et al. Image destriping method based on wavelet transform. Remote Sensing Technology and Application, 2015, 30(6): 1168-1175.

4. Jun Shi, Naitong Zhang and Xiaoping Liu. A novel fractional wavelet transform and its applications. Science China, 2012, 55(6): 1270-1279.

5. Venkatesh YV, Kumar Raja S and Ramya N. Multiple contour extraction from gray level images using an artificial neural network. IEEE Trans on Image Processing, 2006, 15(4): 892-899.

6. Zhiheng Zhou, Delu Zeng and Shengli Xie. RBF neural network and active circles based algorithm for contours extraction. Progress in Natural Science, 2007, 17(6): 681-686. 